\title{
SAÚDE, DOENÇA E INOVAÇÃO TECNOLÓGICA
}

\author{
H. Maria Dutilh Novaes* \\ Ricardo Lafetá Novaes*
}

\begin{abstract}
RESUMO: As inovações tecnológicas em medicina são freqüentemente introduzidas, nos debates que tratam dos processos de saúde/doença existentes em populações determinadas, e das formas de intervenção possíveis por parte da atenção médica, de duas formas polares e antagônicas: ou são as principais responsáveis pelos insucessos, ou são as únicas garantias da efetividade médica. Neste texto argumenta-se, lançando mão de exemplos retirados da análise da presença das doenças cardiovasculares nas populações ao longo do tempo, que é possivel, e desejável, que sejam desenvolvidas atitudes e práticas mais diferenciadas em relação a elas, em que os conhecimentos científicos e técnicos específicos, assentados na individualidade biológica, possam estar melhor articulados com as necessidades e objetivos colocados para os sistemas de saúde, na sua inserção social, política, econômica e cultural. As diversas perspectivas possiveis de serem adotadas para a avaliação nos sistemas e serviços de saúde, que têm tido um desenvolvimento crescente nas conjunturas de "crise na saúde", ainda que não possam negar a sua origem de prática racionalizadora, estão apontando para um real reconhecimento da necessidade de uma maior reflexão no interior das próprias práticas médicas sobre as premissas que as sustentam, e de uma melhor compreensão das formas possiveis para as sociedades hoje vivenciarem e administrarem, de uma forma mais transparente e menos dogmática, os conflitos inevitáveis entre os interesses individuais e as necessidades coletivas.
\end{abstract}

$\mathrm{Na}$ década de 70 verificou-se, na maioria dos paises (incluindo o Brasil) a mais significativa e jamais observada expansão dos gastos em atenção médica(1). Com menor intensidade, este fenômeno persistiu nos anos '80

\footnotetext{
* Professores Doutores do Departamento de Medicina Preventiva da Faculdade de Medicina da Universidade de São Paulo
} 
apresentando, todavia, uma maior variabilidade nas tendências, detectada através de comparações internacionais. Para o Brasil, particularmente nos últimos anos da "década perdida" e início dos anos '90, os dados disponiveis são de difícil análise em decorrência das reestruturações pelas quais vêm passando o financiamento e organização do sistema de atenção médica(2).

Os fatores determinantes das diversas e sempre complexas conjunturas sociais encontram-se razoavelmente identificados. Fundamentalmente de natureza econômica, articulam-se com aqueles político-sociais, ai incluídas especificidades de ordem cientifica, técnica, profissional e cultural que se desdobram, também, em questões éticas e morais. Por isso, as análises e interpretações dai decorrentes têm sido múltiplas e variadas, donde decorre a permanente necessidade de novos estudos e investigações. A reflexão que aqui se pretende desenvolver assenta-se em um recorte bastante específico, qual seja, o modo pelos qual as inovações tecnológicas têm sido introduzidas em estudos que, dimensionando saúde e doença nos individuos e na sociedade, relacionam-as com as formas de intervenção que visam aumentar a saúde e diminuir a doença. $O$ aumento de uma e a diminuição de outra, são resultados freqüentemente tomados como equivalentes mas que, na realidade, referem-se a objetos bastante distintos, constituindo-se em um primeiro problema quando se busca analisar a participação das inovações tecnológicas médicas na obtenção daqueles objetivos.

Problema propriamente conceitual pois que se refere ao significado de expressões, de certa forma corriqueiras, como saúde e doença. Para efeito das considerações aqui contidas, associa-se o termo saúde à idéia de "bem estar" e o de doença àquela de "sofrimento". Se esta última é passivel de uma mais fácil apreensão quantitativa, esta possibilidade não é tão evidente em relação à 
primeira, dado que fatores de ordem subjetiva jogam ai um papel altamente expressivo. Nessa perspectiva, estudos têm sido realizados visando dimensionar técnicas e resultados, associando uns aos outros.

Em uma primeira abordagem das discussões travadas no interior de tais estudos, verifica-se a existência de posições polares que, de certa forma, enrijecem o debate. Ou se atribui ao desenvolvimento tecnológico em medicina um caráter essencialmente positivo ou, ao contrário, representaria ele tão somente interesses extra-médicos, contrários à saúde das populações. No primeiro caso, a tecnologia sempre contribui para diminuir as doenças e, por conseqüência, melhorar as condições individuais e coletivas de saúde. Neste sentido e identificado como o melhor caminho, o desenvolvimento tecnológico conteria, em si, plenas justificativas para todos os possíveis gastos dele decorrentes. No polo oposto, a tecnologia se constituiria tão somente em mais um momento privilegiado da realização de lucros para o capital, impedindo que medidas eficazes sejam tomadas para a consecução do objetivo desejado, qual seja, saúde plena e para todos.

Posturas rígidas, obviamente, encaminham para oposições entre um dever ser e um dever não ser que limitam a compreensão da contribuição das inovações tecnológicas em medicina. Assim, e tendo por objetivo uma melhor argumentação, tomam-se aqui como exemplo concreto as doenças cárdiovasculares, na perspectiva de seu comportamento epidemiológico e dos "tratamentos" adotados nas sociedades contemporâneas. Juntamente com os cânceres, estas doenças dominam o grupo das crônico-degenerativas, em grande parte responsáveis pela progressiva elevação dos gastos em saúde e pela crescente incorporação e utilização de inovações tecnológicas nos serviços médicos $(3,4)$. 
O Brasil, quando comparado com paises mais desenvolvidos ( em parte em decorrência de sua estrutura etária), apresenta taxas mais baixas de mortalidade por doenças do aparelho circulatório. No entanto, e com importantes diferenças regionais, elas já representam, para o conjunto de todas as idades, a mais elevada mortalidade proporcional por grupo de causas. Neste grupo, as doenças cérebro-vasculares apresentam um predomínio sobre as doenças isquêmicas do coração, ao contrário do que ocorre na maioria dos países desenvolvidos(5).

A etiopatologia das doenças do aparelho circulatório é bastante complexa, estando já identificados uma série de fatores de risco sem que, cada um deles isoladamente, possa ser considerado causa necessária e suficiente para 0 surgimento da doença em um individuo específico. Entre eles, são considerados como os mais importantes, os seguintes: idade acima de 40 anos, sexo masculino, hipertensão arterial, hábito de fumar, hipercolesterolemia (principalmente da fração das lipoproteínas de baixa densidade - LDL) e diabetes. Como fatores associados, aponta-se: excesso de peso, vida sedentária, história familiar de doença cardiaca antes dos 65 anos, tipo de personalidade, "stress" e consumo elevado de álcool(6).

Assim listados, estes fatores de risco mostram naturezas muito diversificadas: são doenças, desvios de uma normalidade estabelecida por parâmetros laboratoriais, hábitos individuais, condições de vida, etc. Ademais, muitos deles se constituem em fatores que se associam na história de vida de individuos específicos, sendo difícil analisá-los isoladamente sem que haja prejuízo para uma apreensão mais refinada da realidade. Pode-se bem perceber as dificuldades conceituais e metodológicas colocadas por este tipo de identificação de 
fatores de risco para estudos deste grupo de doenças, tanto nos indivíduos como na coletividade.

Um elemento adicional tem se agregado a esta problemática: na maioria dos países desenvolvidos, a partir dos anos '70 e mais intensamente nos ' 80 , os coeficientes de mortalidade por doenças cárdio-circulatórias têm apresentado uma constante tendência de declínio. Nos Estados Unidos houve uma queda de $40 \% \mathrm{em}$ relação à taxa mais elevada que por último se observou. Suécia, Austria e Alemanha têm apresentado uma certa estabilidade, enquanto países da Europa Oriental mostram um aumento dos coeficientes por doenças coronarianas $(3,7)$.

No Brasil, para o Município de São Paulo, pôde ser detectada uma tendência de queda dos coeficientes de mortalidade por doenças do aparelho circulatórios, com a seguinte diferenciação: a partir de 1970 para as cerebrovasculares e desde 1976 para aquelas cardiovasculares, em adultos maiores de 20 anos e de ambos os sexos( $(8,9)$. Ressalte-se que este comportamento ainda não é generalizável para todas as regiões do país(10).

Estas tendências mais recentes indicam um certa reversão de uma situação que, para efeito de uma periodização simplificada, tem uma acentuação mais expressiva no início do século XX. As explicações para o observado aumento da mortalidade por estas doenças são de ordens variadas: a) o envelhecimento da população em decorrência de queda da mortalidade infantil e da natalidade e a manifestação de um processo biológico inevitável devido a uma maior duração da vida; b) mudanças nas condições de vida das populações, decorrentes de um certo tipo de desenvolvimento econômico e social - urbanização, vida sedentária, condições de trabalho mais propícias a tensões e desgaste psíquico, modificações de hábitos alimentares, expansão do consumo de tabaco e bebidas alcoólicas; $c$ ) 
mudanças introduzidas na nomenclatura médica e nas condições em que se desenvolvem os processos diagnósticos, fazendo com que idênticos processos patológicos recebam, ou não, diagnósticos com denominações e registros variados, cujos efeitos manifestam-se nas estatísticas de mortalidade, variáveis e não uniformes(11).

À luz das considerações acima, como interpretar as recentes quedas da mortalidade por doenças cárdio-vasculares? Houve diminuição também na morbidade ou trata-se de um fenômeno restrito à mortalidade?

É de se esperar que uma queda dos coeficientes de morbidade esteja associada a modificações dos fatores considerados de risco. No caso das doenças cárdio-vasculares são mencionadas as seguintes alterações: diminuição da freqüência do hábito de fumar, maior valorização da atividade física, modificações nas dietas, diagnósticos mais precoces e melhor controle e acompanhamento dos doentes hipertensos e diabéticos, maior vigilância em relação a alterações detectadas laboratorialmente, como niveis de colesterol e LDL, por exemplo. No entanto, os estudos nesta área apresentam uma persistente dificuldade em permitir a generalização dos fenômenos observados em grupos populacionais selecionados (restritos e específicos) para todas as camadas sociais, nas diversas conjunturas. $(12,13) \dot{E}$ que, para muitas destas situações, não se consegue obter elementos suficientes que permitam confirmar modificações substanciais nas condições gerais de vida associadas a muitos dos fatores de risco inicialmente identificados como geradores destas doenças.

Ganham força, neste momento, os que argumentam que a queda da mortalidade por doenças cardiovasculares deve-se, em grande parte, à diminuição de sua letalidade. Apresentam-se como elementos explicativos as grandes 
transformações ocorridas nos processos diagnóstico e terapêutico (a partir da década de '60) tais como: desenvolvimento e expansão das cineangiocoronariografias, da ecocardiografia e de outros métodos cada vez menos invasivos e capazes de produzir imagens e registros gráficos muito precisos. Quanto à terapêutica, ressalta-se a introdução de novas técnicas cirúrgicas ("pontes" coronarianas e transplantes cardíacos), procedimentos "à distância" (raio laser), expansão e aprimoramento do cuidado intensivo em unidades coronarianas, desenvolvimento de novas drogas. Explicações, como se vê, assentadas no desenvolvimento tecnológico verificados na medicina.

Constituindo-se em novas ofertas criadoras de demandas, as inovações tecnológicas configuram um mercado que necessita ser visto mais de perto, principalmente no que diz respeito ao ritmo e intensidade de sua real incorporação e utilização. Em outros termos, trata-se de verificar em que medida uma técnica disponivel torna-se um procedimento disseminado e rotineiro e, ainda, os eventuais efeitos de sua utilização. Abordando a questão pelo ângulo dos gastos estima-se que, na década de ' 80 , os Estados Unidos despenderam, aproximadamente, $25 \%$ de seu orçamento em saúde (algo em torno de $11 \%$ do seu nada desprezível Produto Interno Bruto - PIB) no atendimento às doenças cárdio-vasculares. Na maior parte dos paises da Europa Ocidental, a proporção relativa ao PIB é menor (entre 6 e 9\%), cabendo desta, uma fatia menor ainda (entre 10 a 20\%) destinada às doenças cárdio-vasculares(14) Desta ótica, não é possivel observar uma associação constante entre o volume de gastos e os níveis dos coeficientes de mortalidade por estas doenças. Significa tal constatação que ainda não se pôde estabelecer relações causais (amplamente aceitas) entre a introdução de específicas práticas e procedimentos médicos e correspondentes quedas na mortalidade por doenças cárdio-vasculares. 
Claro está que uma associação ainda não demonstrada não resulta, necessariamente, em sua invalidação. O problema expressa a persistência de dificuldades conceituais e metodológicas quando se busca investigar nexos causais entre fenômenos e práticas de ordens diversas, tais como os eventos biológicos, a complexidade da organização social e tecnologias médicas específicas. Neste sentido $e$ independentemente do grau de confiança depositado na possivel efetividade dos procedimentos médicos, constata-se a divisão de opiniões entre os estudiosos quanto à possibilidade de superação dos dilemas identificados nas investigações atuais. Donde o surgimento de variadas propostas de estudos, que buscam alternativas para resolver os impasses conceituais e metodológicos acima apontados.

No Brasil, o reconhecimento destes problemas desenvolve-se de maneira ainda incipiente. Uma primeira dificuldade a ser superada refere-se à qualidade das informações disponíveis. Sérios problemas são identificados quando se buscam dados confiáveis sobre estatísticas de morbidade produzidas pelos serviços de saúde (inclusive hospitalares) e sobre dispêndios realizados, discriminados por diagnósticos ou por procedimentos. No contexto do sistema previdenciário brasileiro (em expansão desde 1975 quanto a volume de atendimentos e custos) verifica-se que, em 1991, as internações por doenças do aparelho circulatório aparecem em terceiro lugar no conjunto de todas as internações (após partos e doenças respiratórias) mas foram responsáveis pelos maiores gastos em assistência médica, por grupos de doenças. ${ }^{15)}$ Todavia, não se consegue correlacionar os procedimentos realizados com os quadros clínicos que os determinaram, inclusive no que diz respeito aos niveis de risco de vida a eles inerentes. As poucas informações disponiveis (oficiais ou resultantes de algumas investigações especificas e localizadas) reforçam a hipótese de que a incorporação 
e utilização de tecnologia médica no Brasil tem sido moldada, quase que exclusivamente, por uma lógica de máxima remuneração econômico-financeira por parte do setor privado, e de descompromisso social e de descontinuidade nas políticas adotadas, por parte do setor público(16). Resulta que poucos pacientes recebem um grande volume de cuidados (às vezes em "excesso" ou inadequados) enquanto muitos outros quase nenhum. Se para alguns até mesmo qualquer tipo de cuidado médico seria desnecessário, não se tem, por outro lado, dimensionado as necessidades de pessoas que não tiveram acesso aos serviços. Assim, e ainda considerando a total anomalia que representam as fraudes aqui existentes, fácil é perceber as dificuldades na identificação de uma lógica clínica instrumentalizando as práticas diagnósticas e terapêuticas, que possa ser correlacionada com resultados de alguma forma mensuráveis.

Condições sociais mais desfavoráveis para a realização das práticas surgem como agravantes das dificuldades inerentes à própria clínica, no que se refere à avaliação de resultados relacionados à sua intervenção. Ciência e arte, a prática clínica guia-se pela objetividade dos conhecimentos existentes, mas realizando-se por juizos e decisões que envolvem marcantes elementos da subjetividade(17). Inúmeros estudos, por exemplo, têm se dedicado a avaliar a aplicação nos serviços dos critérios clínicos recomendados de indicação de procedimentos diagnósticos e terapêuticos, relacionando-os com o uso corrente efetivamente encontrado(18). De forma recorrente, em muitos casos (as doenças cárdio-vasculares têm sido um dos seus objetos preferenciais), observa-se uma tendência de super-utilização.

Representa tal situação apenas um desvio de uma melhor condição

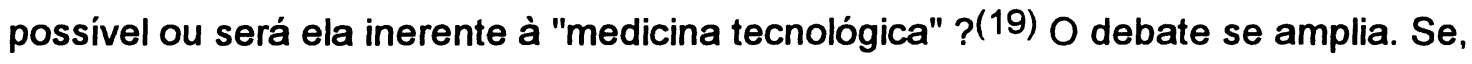


anteriormente e de um lado, encontravam-se aqueles que atribuiam à medicina uma contribuição apenas marginal na determinação dos niveis de saúde dos individuos e das populações e, de outro, os que nela vêm a "salvação" , novos atores entram em cena. Para melhor compreender a nova dimensão deste debate, algumas questões devem ser recuperadas.

Após a Segunda Guerra Mundial, uma das características do desenvolvimento econômico dos paises capitalistas centrais foi a expansão da oferta de serviços médicos e da industria de medicamentos e equipamentos médico-hospitalares. Nos Estados Unidos, em particular, os gastos com saúde tiveram, por várias décadas, um crescimento anual duas vezes maior que o da economia como um todo. De um ponto de vista econômico, político e social, esta situação foi considerada positiva até a década de ' 70 , quando apareceram os primeiros sinais de que setores da sociedade americana e pelos mais diversos motivos, não mais aceitavam incondicionalmente uma tal expansão. Acreditavam que, incontrolável, seria ela desestabilizadora para o conjunto da economia e da sociedade.

Vários instrumentos burocráticos e administrativos foram criados, buscando introduzir critérios de eficácia, efetividade e necessidades nos processos de desenvolvimento tecnológico de produtos específicos. Da certificação de qualidade, da demonstração de eficácia, segurança e necessidade em relação à distribuição territorial, dependeria a permanência do produto no mercado. Todavia, estes mecanismos de controle pouco têm influido nas características gerais do processo técnico-industrial dado que todo novo produto tem tido um difusão relativamente rápida e intensa(20). Resulta que o efeito mais sensível desta pretensão de controle tenha sido, talvez, o de uma sensibilização mais ampliada da 
sociedade para a questão da qualidade dos serviços médicos e uma menor tolerância para com as "aberrações". É inegável, todavia, que os gastos em saúde transformaram-se em importante fato político, exigindo que o Estado responda aos vários grupos de pressão, ainda que, em diversos casos, apenas formalmente.

Uma das exigências é, sem dúvida, a formulação de políticas específicas. Para aquelas "tecnológicas", tem se buscado referências e balizamentos em resultados obtidos por pesquisas médicas. Todavia, os "ensaios clínicos", por exemplo, não têm conseguido oferecer todas as respostas necessárias, ainda que continuem como "ponto de partida" dado que, se não conseguem demonstrar a eficácia de uma determinada técnica, podem decidir pelo seu abandono. Assim, e verificada a limitação deste tipo de estudo, novos parâmetros são introduzidos na avaliação dos procedimentos médicos. Entre os mais frequentes estão as análises de custo benefício, custo efetividade e custo utilidade, estudos epidemiológicos, abordagens "administrativas", sociológicas e psicológicas.

Ocorre que a aplicação de conceitos utilizados nestas análises, essencialmente voltados para fenômenos coletivos, são de difícil individualização. Introduzir conceitos como benefício, utilidade e efetividade na compreensão do processo saúde/doença em indivíduos específicos tem se revelado uma tarefa extremamente complexa, pois estas noções dependem diretamente do valor social atribuído à cada vida humana nos diferentes grupos sociais (jovem ou velho, patrão ou operário, branco ou negro etc.) face a um risco mais evidente e imediato de morte. E mais: hoje, não basta estar vivo. É preciso haver alguma qualidade nesta vida. 
Qualidade de vida é um exigência que tem se encontrado na base de todos os movimentos sociais que procuram obter e garantir direitos à saúde, educação, trabalho, lazer etc. Se hoje parece haver um consenso sobre quais seriam os direitos básicos do cidadão e relativamente mais simples definir condições mínimas que caracterizariam seu reconhecimento, é preciso não esquecer as continuas redefinições decorrentes de especificos processos políticos e econômico-sociais de cada pais. Nesse sentido, não se pode pensar em definições e condições fixas e universais, a-históricas $(21)$.

Nos paises desenvolvidos, onde a maioria da população já tem seus direitos minimamente atendidos e vive cada vez mais, têm surgido problemas que questionam até mesmo valores tidos como intocáveis para as sociedades cristãs ocidentais: é sempre bom viver e viver o máximo possivel ? "Viver sempre mais" não significa outra coisa que prolongar o processo de degenerescência biológica acentuando, coletivamente, suas características? Tratar as doenças próprias do envelhecimento ocupa, atualmente, uma parte majoritária dos gastos em saúde, com tendência crescente. Neste contexto surgem, por exemplo, questões relacionadas ao "direito à morte" de cancerosos terminais, à recusa de tratamentos que, prolongando a vida, mantêm os pacientes inválidos ou padecendo de dores permanentes. De outro lado, situam-se questões relativas a políticas restritivas como as que querem, por exemplo, interditar hemodiálises e transplantes para pacientes com mais de 65 anos de idade ${ }^{(22)}$.

Estes fatos demonstram, mais uma vez, que o "quanto" e o "como" as sociedades - mesmo as mais desenvolvidas econômica e socialmente - querem e podem gastar com atenção médica passam por complexos processos de redefinições. Não se trata mais, apenas, da presença e participação de 
profissionais de saúde e de interesses econômicos mais imediatos de empresas produtoras de bens e serviços. Trata-se, sim, da interveniência de interesses diversificados, políticos, sociais, científicos e culturais.

Mesmo em um país como o Brasil, onde os gastos em saúde são evidente e clamorosamente insuficientes e a iniqüidade do sistema de saúde reflete profundas desigualdades sociais, dificuldades semelhantes fazem-se presentes. Há uma persistente e crescente demanda por serviços médicos, mesmo diante de circunstâncias em que outros tipos de investimentos poderiam, eventualmente, resultar em uma melhor condição geral de saúde da população. Poder ser diagnosticado ou tratado por uma das "conquistas" da medicina tornou-se um ideal a ser alcançado por todos, embora atingido apenas por alguns poucos invejados "eleitos". Não se trata mais de uma mera imposição do "complexo médicoindustrial" que violenta os reais desejos da população, mas de fenômenos muito mais multifacetados.

Uma pergunta pode, então, ser formulada: a partir da compreensão que se vai elaborando, que mudanças podem ser propostas relativamente aos gastos excessivos e/ou inadequados em tecnologias de todo tipo ? E mais, que mudanças devem ser operadas nas demandas por uma maior qualidade nos serviços ? Isso porque, vários "culpados" têm sido identificados nesta "nova" situação, como se pode observar na literatura especializada: consumidores equivocados e iludidos, médicos interesseiros ou "irracionais", sistemas de prestação de serviços rígidos, auto-referidos e injustos, insuficiência de conhecimentos científicos e tecnológicos, a fria lógica do capital e outros mais passiveis de identificação. Todos estes fatores, certamente, são reais e participam, com maior ou menor intensidade, na conformação dos processos de produção, difusão e utilização de inovações 
tecnológicas médicas. Mas, para melhor compreendê-los, convém analisá-los sob uma ótica mais abrangente, necessária, inclusive, para se poder visualizar as transformações possiveis.

Faz-se necessário, de início, identificar quais são as novidades entre esses "culpados", e a sua especificidade para a prática médica. Em primeiro lugar, consumidores sempre fomos, no sentido de que desejos e necessidades são uma constante humana, principalmente no que se refere à atenuação ou eliminação da dor, do sofrimento, da doença. Claro está que esta busca não encontra respostas tão somente nos serviços médicos. No entanto, e de certa forma, têm sido eles eleitos locus produtor privilegiado de soluções almejadas, atribuindo-se-lhes até mesmo o exercício arbitrário de um poder, como se externo fosse à vontade dos homens socialmente organizados. A atenção à saúde é um dos elementos propiciadores da felicidade buscada pelo homens e, por isso, não pode configurarse como a verdadeira e exclusiva panacéia...

Em segundo lugar, médicos "interesseiros", "irracionais" e "incompetentes" não se constituem propriamente em novidade histórica. Não é este "diagnóstico", apenas, que determina a retirada da responsabilidade atribuida a este profissional, mesmo porque dele necessitamos e ainda não se pôde inventar um seu substituto, fabricado para agir em estrito acordo com os mais altos principios éticos e morais. Isto porque o juízo expresso em um diagnóstico e em uma conduta terapêutica não é, jamais, "inocente". Já se viu, a carga subjetiva presente no encontro médico/paciente (sujeito que sabe/sujeito que sofre, sujeito que decide/sujeito que se submete) não permite que a medicina seja uma ciência exata, e ela não tem como não reconhecê-lo apesar de, eventualmente, lamentá-lo. Com estas características, a medicina será sempre aquela historicamente possivel 
e que se realiza em contextos determinados, e a maior presença das inovações tecnológicas e especialização profissional não parecem ter modificado estas características básicas do agir médico.

Os sistemas de prestação de serviços à saúde, em geral rígidos, autoreferidos (como também o são também aqueles educacionais, judiciários etc.) refletem, em seu conjunto, a (in)justiça social presente nas diversas conjunturas. No Brasil vive-se, sem dúvida, neste momento de agilização de nossa persistente e grave crise econômica e política, uma intensificação dos sempre presentes mecanismos de exclusão postos mais a claro, talvez, pela ampliação do espaço de reivindicações, de esforços mais acentuados na luta pela construção de uma nova cidadania. A questão da qualidade encontra, assim, um terreno mais propício para seu florescimento, em todos os setores.

Em relação à proclamada insuficiência de conhecimentos científicos e tecnológicos como causa dos problemas identificados, não é demais lembrar que esta insuficiência é parte inerente ao desenvolvimento científico, que gera, ele mesmo, o deslocamento contínuo de suas próprias fronteiras.

Finalmente, o "lucro a qualquer preço" e que só é possivel através de um consumo crescente de produtos sempre novos, é a mola do sistema econômico hoje mundialmente hegemônico, construindo interesses que the são específicos inclusive, no resguardo de seus objetivos, através da introdução de técnicas que garantam eficácia, qualidade e segurança de seus produtos. Pensar este processo como um atributo gerado exclusivamente no interior da prática médica, empobrece a sua compreensão geral e a identificação das suas especificidades. 
Sendo assim, podemos considerar que nunca existiu um passado, nem haverá um futuro, sem problemas, sem conflitos, sem incertezas: não houve, nem haverá uma saúde plena e eterna para o homem, e demonstrar que esta vem piorando inexoravelmente, pela atuação especifica e intencional da medicina, não se constitui em uma tarefa fácil. Não terá, justamente, a maior visibilidade e transcendência das questões da saúde, e da presença das doenças, para as sociedades contemporâneas, contribuido também para o que agora nos parece às vezes excessivo e desagregador?

Nesta perspectiva, é possivel considerar as inovações tecnológicas na medicina (e as suas condições de incorporação e utilização) como elementos construidos pelas condições históricas gerais articuladas aos processos específicos do saber e das práticas médicos, não se constituindo em benefícios ou malefícios em si. É fato que as inovações tecnológicas em medicina vieram para ficar, cabendo avaliá-las permanentemente, para que os resultados do seu uso possam ser os mais favoráveis possiveis, necessariamente integrados às contínuas transformações por que passam os sistemas de saúde.

É no reconhecimento de suas articulações gerais mas, também, na delimitação de suas especificidades, que podem dar sua contribuição os estudos que se desenvolvem, não por acaso, a partir da década de '70, conhecidos como de avaliação econômica, avaliação tecnológica e avaliação de qualidade de serviços (23). O exemplo das doenças cárdio-vasculares, aqui utilizado, evidencia que estes enfoques não trazem, em si, respostas definitivas para a problemática hoje enfrentada. Todavia, podem contribuir para que opções específicas sejam feitas de forma mais clara, permitindo uma maior participação da sociedade na definição de suas perdas e possiveis ganhos. 


\section{REFERÊNCIAS BIBLIOGRÁFICAS}

1. CREDES. Programme Éco Santé OCDE. Paris, CREDES/OCDE, 1993.

2. MEDICI,A.C. Gastos com saúde nas três esferas de govêrno:1980-1990 - São Paulo, IEPS/FUNDAP, 1993.[mimeografado]

3. Mc KINLAY,J.B.; Mc KINLAY,S.M.; BEAGLEHOLE,R. A review of the evidence concerning the impact of medical measures on recent mortality and morbidity in the United States. Int. J. Hith Serv., 19:181-208,1989.

4. LOPEZ,A.D. Assessing the burden of mortality from cardiovascular diseasesWd Hlth Statist. Quart.,46:91-6, 1993.

5. MINISTÉRIO DA SAÚDE. INAMPS: doenças crônico-degenerativas: evolução e tendências atuais. Brasilia, 1988.

6. WYNGAARDEN,J.B. \& SMITH,L.H. Cecil's textbook of medicine. Philadelphia, W.B. Saunders Company, 1985. v.1, p.150-367: Cardiovascular Diseases,

7. BEAGLEHOLE,R. International trends in coronary heart disease: mortality, morbidity and risk factors Epidemiol. Rev., 12:1-15,1990.

8. LOLIO,C.A. \& LAURENTI,R. Mortalidade por doença isquêmica do coração no Município de São Paulo: evolução de 1950 a 1981 e mudanças recentes na tendência- Arq. Bras. Cardiol., 트:153-6,1986.

9. LOLIO,C.A. \& LAURENTI,R. Tendência de mortalidade nas doenças cerebrovasculares em adultos maiores de 20 anos de idade no Município de São Paulo, Brasil, 1950-1981- Rev. Saúde Pública.,S.Paulo.,20:343346,1986.

10. LESSA,I. Epidemiologia, assistência médica e impacto econômico e social das doenças cardiovasculares e do diabetes mellitus. Inf. Epidemiol. SUS, 2(4):5-20,1993.

11. BOUVIER-COLLE,M.H.; VALLIN,J.; HATTON,F. Mortalité et causes de décès en France, Paris, DOIN/ INSERM, 1990. 
12. ELUF NETO,J.; LOTUFO,P.A.; LOLIO,C.A. Tratamento da hipertensão e declínio da mortalidade por acidentes vasculares cerebrais- Rev. Saúde Pública,S.Paulo, 24:332-6,1990.

13. ALMEIDA FILHO,N. Epidemiologia sem números - Rio de Janeiro, Campus, 1989.

14. HARRISON,D.C. Cost-containment in medicine: why cardiology. Am.J. Cardiol.,56(Suppl.):10C-15C,1985. (suppl.)

15. BUSS,P.M. Assistência hospitalar no Brasil (1984-1991): uma análise preliminar no sistema de informação hospitalar do SUS. Inf. Epidem. SUS,2(2):5$42,1993$.

16. NOVAES, H.M.D. Processo de desenvolvimento tecnológico em saúde: demanda e uso de tecnologia: o consumo hospitalar no Município de São Paulo. São Paulo, 1990.[mimeografado]

17. NOVAES,H.M.D. Diagnosticar e classificar: o limite do olhar. São Paulo, 1987.[Tese de Doutoramento - Faculdade de Medicina da USP]

18. MCPHERSON,K. Variations entre pays des pratiques médicales In: Les systèmes de santé: a la recherche d'éfficacité Paris, OCDE, 1990. p. 17-30.

19. SCHRAIBER,L.B. O médico e seu trabalho: limites da liberdade. São Paulo, Editora Hucitec, 1993.

20. JACOBY,I. Update on assessment activities- United States perspective. Int. J. Tech. Assess. Hith Care, 4:95- 105,1988.

21. COHN,A., NUNES,E., JACOBI,P.R.; KARSCH,V.S. A saúde como direito e como serviço. São Paulo, Cortez Editora/ CEDEC, 1991.

22. STOCKING,B. Expensive health technologies: regulatory and administrative mechanisms in Europe. Oxford, Oxford University Press, 1988.

23. BANTA,H.D. \& LUCE,B.R. Health care technology and its assessment: an international perspective - Oxford, Oxford University Press, 1993. 